\section{CAPAP in acute pancreatitis: just another marker or real progress?}

There are no pathognomonic symptoms in acute pancreatitis. In reaching a diagnosis the patient's history, physical findings, and serum amylase or lipase concentrations have to be considered. Serum amylase is one of the oldest clinically useful laboratory tests, having been around for almost 70 years. ${ }^{1}$ However, too great a reliance on amylase or lipase will lead to underdiagnosis owing to the lack of sensitivity and specificity. ${ }^{2}$

Up to $20 \%$ of patients with acute pancreatitis run a severe clinical course and these patients must be identified as quickly as possible. The "gold standard" for staging patients with acute pancreatitis is dynamic contrast enhanced computed tomography. ${ }^{3}$ Disease severity, however, may only become apparent at laparotomy (or necropsy). Operative intervention is not needed in uncomplicated cases and should be avoided if at all posssible in severe acute pancreatitis. Researchers have also attempted to differentiate between mild and severe forms of acute pancreatitis using so-called indicators of necrosis in blood or urine. Examples of these parameters are C-reactive protein (CRP), PMN elastase, phospholipase $\mathrm{A}_{2}$, antiproteases, and cytokines. ${ }^{4-8} \mathrm{CRP}$ is the most useful in clinical practice, but its use is hampered by the fact that the differentiation between mild and severe disease is best three to four days after onset. Some of the others still have methodological problems, or are only used experimentally, or their measurement is too time consuming, or too expensive.

Recently a Finish group have reported on complex formation between trypsin 2 and $\alpha_{1}$-antitrypsin in acute pancreatitis. ${ }^{9}$ They showed that the serum concentrations of the complex between trypsin 2 and $\alpha_{1}$-antitrypsin was increased in all 28 cases of severe pancreatitis but in only three of the 82 with mild pancreatitis. This verifies the results of earlier studies on the pathophysiology of acute pancreatits, which indicated that it is the activated trypsin in the pancreas and its vicinity that causes the inflammatory reaction. ${ }^{10}$ As the reaction is local, it is impossible to measure, and therefore one must measure traces of trypsin activity in blood or urine. One way to do this is to measure the small amounts of active trypsin which bind to protease inhibitors making their way to the systemic circulation (trypsin $2-\alpha_{1}$-antitrypsin complex). This is technically possible today and there is good evidence for a correlation between the concentration of these protease inhibitors and disease severity. ${ }^{911}$ However, it is still not known whether these complexes are present in other acute intra-abdominal inflammatory conditions, and the method is not yet suitable for emergency analysis.

Another recently described method to facilitate the diagnosis of acute pancreatitis is the measurement of trypsinogen 2 (anodal trypsinogen) in urine. ${ }^{12}$ Its concentration in urine correlates strongly with disease severity, in contrast to cathodal trypsinogen and amylase. The explanation is probably that these enzymes are normally excreted via glomerular filtration in the primary urine and at least $90 \%$ are degradated in the kidney tubuli. In acute pancreatitis the tubuli are heavily loaded by an increased filtration of large amounts of pancreatic enzymes with the concomitant occurrence of renal insufficiency. ${ }^{13}$ Why this affects the anodal trypsin to a higher degree than the other enzymes is not known, but provides a good basis for fast immunological methods.

Another way of detecting trypsinogen activation is to measure the peptide that is cleaved from trypsinogen during activation to trypsin, a method first examined by Hermon-Taylor et al. ${ }^{14}$ The activation peptide of trypsinogen, TAP, can be measured in urinary samples using a radioimmunoassay. There is good evidence for a correlation between the TAP concentration and disease severity. However, it has not been proved that TAP is derived from inflamed pancreatic tissue, and antibody to TAP is difficult to produce as it is so small (just five amino acids). Even though the potential of TAP has been noticed for almost a decade now, there is still a lack of clinical data proving its superiority over CRP.

Appelros et al, in this issue (see page 97), present theories that are similar to those of Hermon-Taylor's group, but they have based their assay on the activation peptide of carboxypeptidase B - that is, CAPAP, instead. This peptide (95 amino acids) is considerably larger than TAP, making its measurement more reliable. Moreover, the CAPAP is stable in both serum and urine, which is an advantage over TAP. The Malmö group has found CAPAP in large concentrations in the urine in cases of acute pancreatitis. This large peptide can be present only if trypsin is activated and procarboxypeptidase B is available as a substrate, and, therefore, is likely to reflect the activation of trypsin. Most interestingly, Appelros et al also showed that there is a strong correlation between the concentrations of CAPAP in serum and urine and the severity of acute pancreatitis. This offers for the first time a reliable staging method in acute pancreatitis because, in contrast to the other markers for the assessment of disease severity, the concentration of CAPAP directly reflects the degree of pancreatic damage. However, as only a few patients with severe acute pancreatitis have been investigated so far using CAPAP analysis, Appelros et al's study should be seen as the first step in the evaluation of this new and interesting parameter. Additionally, the specificity of this test in serum and urine has yet to be defined.

Soon we may be able to diagnose and stage acute pancreatitis using a single test, and CAPAP analysis is a promising candidate.

M W BÜCHLER W UHL

Å ANDRÉN-SANDBERG

Correspondence to: Markus W Büchler, MD, University Hospital of Bern, Department of Visceral and Transplatation Surgery, Inselspital, CH-3010 Bern, Switzerland

1 Elman R, Arneson N, Graham E. Value of blood amylase estimation in the diagnosis of pancreatic disease. A clinical study. Arch Surg 1929;19:943-5.

2 Lankisch PG, Schirren CA, Kunze E. Undetected fatal acute pancreatitis: why is the disease so frequently overlooked? $A m \quad \mathcal{F}$ Gastroenterol 1991;86:322-6.

3 Balthazar EJ. CT diagnosis and staging of acute pancreatitis. Radiol Clin North Am 1989;27:19-37

4 Büchler M, Malfertheiner P, Schoetensack C, et al. Sensitivity of antiproteases, complement factors and C-reactive protein in detecting pancreatic necrosis. Results of a prospective clinical study. Int $\mathcal{F}$ Pancreatol 1986;1:227-35. 
5 Wilson C, Heads A, Shenkin A, et al. C-reactive protein, antiproteases and complement factors as objective markers of severity in acute pancreatitis. Brf Surg 1989,76:177-81.

6 Uhl W, Büchler M, Malfertheiner P, et al. PMN-Elastase in comparison with $\mathrm{CRP}$, antiproteases and LDH as indicators of necrosis in human acute pancreatitis. Pancreas 1991;6:253-9.

7 Büchler M, Malfertheiner P, Schädlich $\mathrm{H}$, et al. Role of phospholipase $\mathrm{A}_{2}$ in human acute pancreatitis. Gastroenterology 1989;97:1921-6.

8 Kusske AM, Rongione AJ, Reber HA. Cytokines and acute pancreatitis. Gastroenterology 1996;110:639-42.

9 Hedström J, Sainio V, Kemppainen E, et al. Serum complex of trypsin 2 and $\alpha_{1}$-antitrypsin as diagnostic and prognostic marker of acute pancreatitis: clinical study in consecutive patients. BMF 1996;313:333-7
10 Ohlsson K, Baldin G, Bohe M, et al. Pancreatic proteases and antiproteases in pancreatic disease; biochemical, pathophysiologic and clinical aspects. in pancreatic disease; biochem

11 Borgström A, Lasson A. Trypsin-alpha-1-protease inhibitor complexes in serum and the clinical course in acute pancreatitis. Scand $\mathcal{f}$ Gastroenterol 1984;19:1119-22.

12 Hedström J, Korvuo A, Kenkinmäki P, et al. Urinary trypsinogen-2 test strip for acute pancreatitis. Lancet 1996;347:729-31.

13 Johnson SG, Ellis CJ, Levitt MD. Mechanism of increased renal clearence of amylase/creatinine in acute pancreatitis. N Engl F Med 1976;295:1214-7.

14 Gudgreon AM, Heath DI, Hurley P, et al. Trypsinogen activation peptides assay in the early prediction of severity of acute pancreatitis. Lancet 1990; 335:4-8.
The study reported on page 17 illustrates some of the frustrations of clinical trials. The rationale for undertaking a prospective trial of the use of ranitidine in gastric cancer was appropriate. A previous study from Tonnesen et $a l^{1}$ had demonstrated a survival benefit using cimetidine in patients with gastric cancer. This group of authors argued that cimetidine had an immunological effect which included inhibition of $T$ suppresser activity and increased interleukin 2 production by lymphocytes. ${ }^{23}$

Ranitidine has a similar effect: the Yorkshire GI tumour group began this study in 1989 to evaluate the potential effect of this drug in a controlled trial treating all stages of gastric cancer. Between 1989 and 1995, 222 patients were recruited. This illustrates one of the frustrations of running trials in gastric cancer. This is a recruitment rate of less than 50 patients per year and is a feature many gastric cancer trials have in common. ${ }^{45}$ The patients were randomised to receive either ranitidine or placebo. The randomisation has worked satisfactorily: the number of patients distributed between the placebo and active treatment groups was equal. The most significant prognostic factor in gastric cancer is stage and the randomisation resulted in an higher proportion of patients in tumour stages I and II randomised to the treatment group compared with the control group $(29 \% v 21 \%)$. It is the patients in stages I and II disease that have the best long term survival. This distribution has had the result of loading the placebo group with those of worse prognosis: stages III and IVa and $b$. The mean length of follow up was 185 days. There was no statistical benefit from treatment. The figures suggest that patients receiving ranitidine may be doing better than those in the placebo group. The authors honestly report that this is not a statistical benefit and this highlights the problem of a randomised controlled trial with insufficient numbers. This is acknowledged by the authors in their discussion.

Previous studies using cytotoxic chemotherapy have generally looked for big effects ( 15 to $20 \%$ improvements). It is felt that large benefits should be achieved as many cytotoxic regimes have significant side effects, or reduce the quality of life in patients with advanced disease. However, this is not the case with ranitidine. This study demonstrates it has been well tolerated and it would be in order to look for a small effect since the quality of life is unlikely to be diminished by taking $\mathrm{H}_{2}$ receptor antagonists: such a study would require 2000 patients. Other studies have been undertaken: the British Stomach Cancer Group have now recruited over 400 patients to a trial of cimetidine. However, even analysis of the combined studies is unlikely to have enough patients to be able to demonstrate a small benefit. The other factor that cannot be stressed too strongly is our inability to recruit significant numbers of patients with gastric cancer into trials.

Hopefully the reorganisation of cancer services and the identification of clinicians specifically interested in gastric cancer will allow valuable studies to be undertaken over a reasonable time period.

Queen Elizabeth Hospital,

J W L FIELDING

Edgbaston,

Birmingham B15 2TH, UK

1 Tonnesen $\mathrm{H}$, Bulow $\mathrm{S}$, Fischerman $\mathrm{K}$, et al. The effect of cimetidine on survival after gastric cancer. Lancet 1988;ii:990-2.

2 Gifford RRM, Tilberg AF. Histamine type 2 receptor antagonist immune modulation. II. Cimetidine and ranitidine increase interleukin 2 production. Surgery 1987;102:242-7.

3 Griswold DE, Alessi S, Badger AM, Poste G, Hanna N. Inhibition of T suppressor cell expression by histamine type 2 receptor antagonists. F Immunol 1984;132:3054-7.

4 Cuschieri A, Fayers P, Fielding JWL, et al. Post-operative morbidity and mortality after D1 and D2 resection for gastric cancer: Preliminary results of the of the MRC randomised controlled surgical trial. Lancet 1996;347: 995-9.

5 Hallissey MT, Dunn JA, Ward LC, Allum WH. The second British Stomach Cancer Group trial of adjuvant radiotherapy and chemotherapy in resectable gastric cancer: two year follow up. Lancet 1994;344:1309-12.
The paper by Kingham and Parker (see page 120) provides a strong take home message regarding the importance of establishing associations between diseases which, although relatively rare, have an impact on the health of a relatively young population. The authors have demonstrated an association between the two disorders and suggest that patients with one disease should be tested for the other. Is this advice justified?

Justification for such targeted screening depends on three things: the robustness of the association; the simplicity and reliability of the diagnostic tests; and the evidence that early detection benefits the patient.

The study aims to make a rigorous assessment of the previously described association between coeliac disease and primary biliary cirrhosis, ${ }^{1}$ and establish relative 
prevalences of the two diseases. Gastroenterologists in South Wales have kept a register of specifically defined gastrointestinal diseases since 1984, using ICD coding from all in- and outpatient and day case attendances in the department of gastroenterology. The population under study is accurately defined by use of postcode and ONS statistics. These show a relatively stable population of approximately 250000 individuals (only $0.2 \%$ population growth and little migration or immigration). Multiple case finding methods are used to identify all possible cases of each disease and inclusion criteria are clearly specified. Possible reasons for underdetection of cases are examined.

The study identified 143 patients with coeliac disease and 67 with primary biliary cirrhosis. Point prevalences in 1995 were 54 per 100000 population for coeliac disease and 20 per 100000 for primary biliary cirrhosis. Four patients were diagnosed with both diseases, giving a point prevalence of 1.6 per 100000 . Those not detected clinically were diagnosed either by investigation of coeliac patients with abnormal liver function tests, or by investigation of patients with primary biliary cirrhosis with features of malabsorption, anaemia, a family history of coeliac disease, or a positive antireticulin antibody test.

This study confirms and defines the association of these two immunologically mediated diseases. The method is a good example of the new trend towards the application of simple epidemiological principles to describe accurately the incidence and prevalence of diseases. ${ }^{2}$ In this study the study period and population are well defined. Criteria for defining each disease are clear and objective. Multiple case finding methods are used. Appropriate, simple statistical methods are used. Flaws and possible causes for underdiagnosis are considered. The prevalence rates are comparable with those found in other studies, both for the individual diseases ${ }^{34}$ and for the rates of disease association.

These figures indicate that around $3 \%$ of patients with coeliac disease may develop or have primary biliary cirrhosis, whereas $6 \%$ of those with primary biliary cirrhosis may develop or have underlying coeliac disease. Possible mechanisms for this association are not discussed in depth. The authors conclude with recommendations for screening for primary biliary cirrhosis in coeliac disease by testing for antimitochondrial antibody and testing for antigliadin antibody in all cases of primary biliary cirrhosis in order to detect coeliac disease.

The proposed screening methods are important as they come close to fulfilling the requirements of any screening programme. These include targeting screening at a high risk population by the use of cheap, simple, acceptable tests, which are sensitive and specific. However, the other criteria that need to be fulfilled are knowledge of the natural history of the diseases involved when detected by screening and proof that intervention favourably affects prognosis. These are more problematic, both in this case and with any screening programme. This is because studies of the natural history of a disease and the efficacy of any treatment modality are rarely performed on the patient cohort diagnosed via a screening programme. Thus, the disease course and response to treatment may be different in screened cases compared with those presenting with symptoms or signs of the disease.

In the case of screening those with coeliac disease for primary biliary cirrhosis, $3 \%$ of patients with coeliac disease may be affected. The antimitochondrial antibody test is both sensitive and specific for identifying cases of primary biliary cirrhosis ${ }^{5}$ and, of course, non-invasive. The natural history is relatively well defined ${ }^{56}$ and double blind, randomised control trials have confirmed the benefit of

\section{Key messages}

Application of epidemiological principles allows study of rare diseases even in relatively small populations and has led to proof of disease association between primary biliary cirrhosis and coeliac disease.

Screening programme requirements are met:

- low symptom group

- high prevalence of disease

- inexpensive, non-invasive, accurate test

- effective treatment

All cases of primary biliary cirrhosis and coeliac disease should be screened for the other disease.

treatment with ursodeoxycholic acid. ${ }^{7}$ The success of liver transplantation in these patients is undisputed. The application of these studies to a screened population is acceptable, as they have included patients diagnosed while in the asymptomatic phase of the illness, who are unlikely to differ notably from a screened population.

The case for screening those with primary biliary cirrhosis for coeliac disease is similarly compelling. Initial testing is non-invasive: antigliadin antibody testing is sensitive, and endoscopic small bowel biopsy is specific. The natural history and increased risk of malignancy (with an excess of lymphomas) in untreated cases is well documented, as is the rapid improvement of nutrition, anaemia and osteoporosis with a gluten free diet. ${ }^{8}$ However, most studies relate to cases presenting with symptoms or complications of coeliac disease. The increased risk of malignancy in an asymptomatic, screened population, and the effect of dietary therapy, is uncertain. However, the correction of malnutrition, anaemia and osteoporosis is of particular importance in primary biliary cirrhosis, as these are all complications of the disease itself and have considerable impact upon both disease outcome and success of transplantation. In addition, those patients undergoing liver transplantation are at increased risk of malignancy (particularly lymphoma) from immunosuppression and thus identification of any potentially correctable risk is of particular merit.

In conclusion, this is an important paper because it has established the true association of coeliac disease and primary biliary cirrhosis and the need for screening for one disease when the other is identified. It serves as a model for the correct application of epidemiological principles to the study of relatively rare diseases. It may also be used as a model to illustrate the importance and potential benefits of a correctly designed screening programme.

Gloucestershire Royal Hospital,

J METCALF

Great Western Road,

Gloucester GL1 3NN, UK

1 Logan RF, Ferguson A, Finlayson ND, et al. Primary biliary cirrhosis and coeliac disease: an association? Lancet 1978; i:230-3.

2 Metcalf JV, Bhopal RS, Howel D, et al. Primary biliary cirrhosis: epidemiology helping the clinician. $B M \mathcal{F} 1996 ; 312: 1181-2$.

3 Metcalf JV, James OFW. Geo-epidemiology of primary biliary cirrhosis. Semin Liver Dis 1997;17:13-22.

4 Bode S, Gudmand-Hoyer E. Incidence and prevalence of adult coeliac disease within a defined geographic area in Denmark. Scand $\mathcal{f}$ Gastroenterol 1996;31:294-9.

5 Metcalf JV, Mitchison HC, Palmer JM, et al. The early natural history of primary biliary cirrhosis. Lancet 1996;348:1399-402.

6 Goudie BM, Burt AD, Macfarlane GJ, et al. Risk factors and prognosis in primary biliary cirrhosis. Am ₹ Gastroenterol 1989;84:713-16

7 Lindor KD, Dickson ER, Baldus WP, et al. Ursodeoxycholic acid in the treatment of primary biliary cirrhosis. Gastroenterology 1994:106:1284-90.

treatment of primary biliary cirrhosis. Gastroenterology 1994;106:1 284-90.

8 Ciacci C, Maurelli L, Klain M, et al. Effects of dietary treatment on bone Am $\mathcal{f}$ Gastroenterol 1997;92:992-6. 